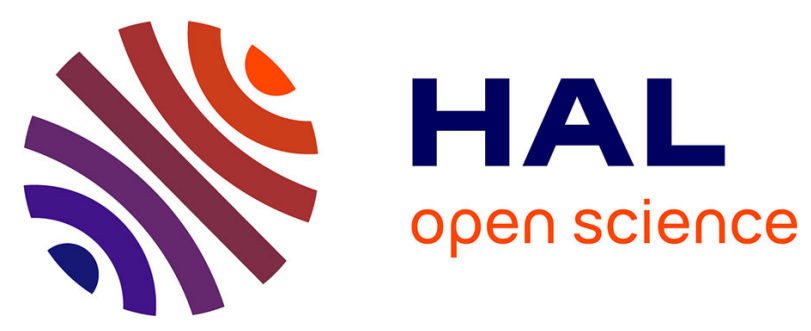

\title{
Computational phenotype prediction of ionizing-radiation-resistant bacteria with a multiple-instance learning model
}

Sabeur Aridhi, Haithem Sghaier, Mondher Maddouri, Engelbert Mephu Nguifo

\section{To cite this version:}

Sabeur Aridhi, Haithem Sghaier, Mondher Maddouri, Engelbert Mephu Nguifo. Computational phenotype prediction of ionizing-radiation-resistant bacteria with a multiple-instance learning model. BioKDD '13 - Proceedings of the 12th International Workshop on Data Mining in Bioinformatics, Aug 2013, Chicago, United States. 10.1145/2500863.2500866 . hal-01819542

\section{HAL Id: hal-01819542 \\ https://inria.hal.science/hal-01819542}

Submitted on 5 Jul 2018

HAL is a multi-disciplinary open access archive for the deposit and dissemination of scientific research documents, whether they are published or not. The documents may come from teaching and research institutions in France or abroad, or from public or private research centers.
L'archive ouverte pluridisciplinaire HAL, est destinée au dépôt et à la diffusion de documents scientifiques de niveau recherche, publiés ou non, émanant des établissements d'enseignement et de recherche français ou étrangers, des laboratoires publics ou privés. 


\section{Computational phenotype prediction of ionizing-radiation-resistant bacteria with a multiple-instance learning model}

\author{
Sabeur Aridhi* \\ CNRS, UMR 6158, LIMOS, \\ F-63173 Aubiere, France. \\ University of Clermont, Blaise \\ Pascal University, LIMOS, BP \\ 10448, F-63000 \\ Clermont-Ferrand, France. \\ University of Tunis El Manar, \\ LIPAH - FST, Academic \\ Campus, Tunis 2092, Tunisia. \\ Mondher Maddouri \\ University of Tunis El Manar, \\ LIPAH - FST, Academic \\ Campus, Tunis 2092, Tunisia.
}

\author{
Haitham Sghaier \\ Unit of Microbiology and \\ Molecular Biology, National \\ Center for Nuclear Sciences \\ and Technologies (CNSTN), \\ Sidi Thabet Technopark,2020 \\ Sidi Thabet, Tunisia
}

\begin{abstract}
Ionizing-radiation-resistant bacteria (IRRB) are important in biotechnology. The use of these bacteria for the treatment of radioactive wastes is determined by their surprising capacity of adaptation to radionuclides and a variety of toxic molecules. In silico methods are unavailable for the purpose of phenotypic prediction and genotype-phenotype relationship discovery. We analyzed basal DNA repair proteins of most known proteomes sequences of IRRB and ionizing-radiation-sensitive bacteria (IRSB) in order to learn a classifier that correctly predicts unseen bacteria. In this work, we formulated the problem of predicting IRRB as a multiple-instance learning (MIL) problem and we proposed a novel approach for predicting IRRB. We used a local alignment technique to measure the similarity between protein sequences to predict ionizing-radiation-resistant bacteria. The first results are satisfactory and provide a MIL-based prediction system that predicts whether a bacterium belongs to IRRB or to IRSB. The proposed system is available online at http://home.isima.fr/irrb/
\end{abstract}

* to whom correspondence should be addressed

Permission to make digital or hard copies of all or part of this work for personal or classroom use is granted without fee provided that copies are not made or distributed for profit or commercial advantage and that copies bear this notice and the full citation on the first page. To copy otherwise, to republish, to post on servers or to redistribute to lists, requires prior specific permission and/or a fee.

BIOKDD ' 13 Chicago, IL, USA

Copyright 2013 ACM 978-1-4503-2327-7 ...\$15.00.

\section{Categories and Subject Descriptors}

J.3 [Computer Applications]: Computational biology

\section{General Terms}

Theory

\section{Keywords}

Phenotypic prediction, ionizing-radiation-resistant bacteria, ionizing-radiation-sensitive bacteria, protein sequences, multiple-instance learning

\section{INTRODUCTION}

Nuclear waste contains a variety of toxic and radioactive substances. The bioremediation of these wastes with pertinent bacteria and low cost is a challenging problem 12, 15. The use of ionizing-radiation-resistant bacteria (IRRB) for the treatment of these radioactive wastes is determined by their surprising capacity of adaptation to radionuclides and to a variety of toxic molecules. To date, genomic databases indicate the presence of thousands of genome projects. However, only a few computational works are available for the purpose of phenotypic prediction discovery that rapidly determines useful genomes for the bioremediation of radioactive wastes [15.

A main idea in this context is that resistance to ionizing radiation and tolerance of desiccation are two complex phenotypes, and suggest that protection and repair mechanisms are complementary in IRRB. In addition, it seems 
that the shared ability of IRRB to survive the damaging effects of ionizing radiation and desiccation is the result of basal DNA repair pathways and that basal DNA repair proteins in IRRB, unlike many of their orthologs in ionizingradiation-sensitive bacteria (IRSB), present a strong ability to effectively repairs damage incurred to DNA.

In this work, we study the basal DNA repair protein of IRRB and IRSB to solve the problem of phenotypic prediction in IRRB. Thus, we consider that each studied bacterium is represented by a set of DNA repair proteins. Due to this fact, we formalize the problem of phenotypic prediction in IRRB as a multiple instance learning problem (MIL) in which bacteria represent bags and repair proteins of each bacterium represent instances.

Many multiple instance learning algorithms have been developed to solve several problems such as predicting types of Protein-Protein Interactions (PPI) 17] and drug activity prediction 4], mainly including Diverse Density 8], Citation-kNN and Bayesian-kNN [16]. Diverse Density (DD) was proposed in 8 as a general framework for solving multi-instance learning problems. The main idea of DD approach is to find a concept point in the feature space that are close to at least one instance from every positive bag and meanwhile far away from instances in negative bags. The optimal concept point is defined as the one with the maximum diversity density, which is a measure of how many different positive bags have instances near the point, and how far the negative instances are away from that point. In [16, the minimum Hausdorff distance was used as the bag-level distance metric, defined as the shortest distance between any two instances from each bag. Using this bag-level distance, we can predict the label of an unseen bag using the $k-N N$ algorithm.

The above cited algorithms use an attribute-value format to represent their data. A most used approach to represent protein sequences in an attribute-value format is to extract motifs that can serve as attributes. Appropriately chosen sequence motifs may reduce noise in the data and indicate active regions of the protein. A protein can then be represented as a set of motifs 2, 13 or as a vector in a vector space spanned by these motifs [14]. However, the use of this technique is not suitable in the context of phenotypic prediction of IRRB. This is due to the fact that the set of proteins of each bag must be represented (in the attribute-value format) with the same set of attributes which is possible only if all extracted motifs from the different bag of proteins are putting together as a unique set of motifs. As the different bags of proteins are processed disjointly, it is necessary to design a novel approach for such case.

In this paper, we propose a MIL approach for predicting IRRB using proteins implicated in basal DNA repair in IRRB. We used a local alignment technique to measure the similarity between protein sequences of the studied bacteria to predict ionizing-radiation-resistant bacteria. To the best of our knowledge, this is the first work which proposes an in silico approach for phenotypic prediction in IRRB.

The remainder of this paper is organized as follows. Section 2 presents the materials and methods used in our study. In Section 3, we describe our experimental techniques and we discuss the obtained results. Concluding points and possible future directions make the body of Section 4

\section{MATERIALS AND METHODS}

\subsection{Terminology and problem formulation}

The task of multiple instance learning (MIL) was coined by Dietterich et al. 3 when they were investigating the problem of drug activity prediction. In multiple-instance learning, the training set is composed of $n$ labeled bags. Each bag in the training set contains $k$ instances and have a bag label $y_{i} \in\{-1,+1\}$. We notice that instances of each bag have labels $y_{i j} \in\{-1,+1\}$, but these values are not known during training. The most common assumption in this field is that a bag is labeled positive if at least one of its instances is positive, which can be expressed as follows:

$$
y_{i}=\max _{j}\left(y_{i j}\right) .
$$

The task of MIL is to learn a classifier from the training set that correctly predicts unseen bags. Although MIL is quite similar to traditional supervised learning, the main difference between the two approaches can be found in the class labels provided by the data. According to the specification given by Dietterich et al. [3], in a traditional setting of machine learning, an object $m$ is represented by a feature vector (an instance) which is associated to a label. However, in a multiple instance setting, each object $m$ may have $k$ various instances denoted $m_{1}, m_{2}, \cdots, m_{k}$. The difference between the traditional setting of machine learning and the multiple instance learning setting can be represented clearly in Figure 1 where the difference between the input objects is shown.
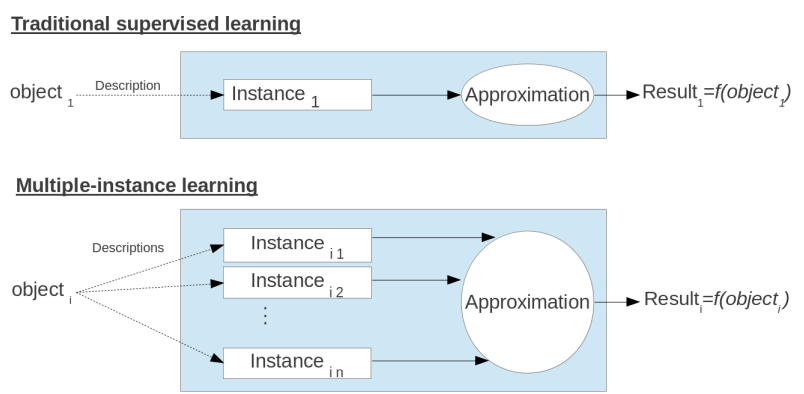

Figure 1: Differences between traditional supervised learning and multiple instance learning.

In our work, we are interested to a specific bacteria family with high radioresistance to ionizing radiation and tolerance of desiccation. This family contains a set of bacteria. Let $D B=\left\{X_{1}, \ldots, X_{n}\right\}$ be a bacteria database. Each bacterium in the database is represented by a set of proteins $X_{i}=\left\{p_{i 1}, \cdots, p_{i k}\right\}$ and belongs to a class label $y_{i}$ with $y_{i}=\{I R R B, I R S B\}$. The problem of phenotypic prediction of IRRB can be seen as a MIL problem in which bacteria represent bags, and basal DNA repair proteins of each bacterium represent instances.

The problem investigated in this work is to learn a multiple-instance classifier in this setting. Given a query bacterium $Q=\left\{p_{1}, \cdots, p_{k}\right\}$, the classifier must use primary structures of basal DNA repair proteins in $Q$ and in each bag of $D B$ to predict the label of $Q$. 
Table 1: Used IRRB and IRSB

\begin{tabular}{|l|l|l|}
\hline ID & Bacterium & Phenotype \\
\hline B1 & Acinetobacter radioresistens SH164 & \\
B2 & Kineococcus radiotolerans SRS30216 & \\
B3 & Methylobacterium radiotolerans JCM 2831 & \\
B4 & Deinococcus maricopensis DSM 21211 & IRRB \\
B5 & Gemmata obscuriglobus UQM 2246 & \\
B6 & Deinococcus proteolyticus MRP & \\
B7 & Truepera radiovictrix DSM 17093 & \\
B8 & Acinetobacter radioresistens SK82 & \\
\hline B9 & Escherichia coli OP50 & \\
B10 & Neisseria gonorrhoeae MS11 & \\
B11 & Neisseria gonorrhoeae PID1 & IRSB \\
B12 & Neisseria gonorrhoeae DGI18 & \\
B13 & Pseudomonas putida S16 & \\
B14 & Thermus thermophilus SG0.5JP17-16 & \\
\hline
\end{tabular}

\subsection{MIL-ALIGN algorithm}

Based on the formalization, we propose the MIL-ALIGN algorithm allowing to predict ionizing-radiation-resistant bacteria. The proposed algorithm focuses on discriminating bags by the use of local alignment technique to measure the similarity between each protein sequence in the query bag and corresponding protein sequence in the different bags of the learning database.

In MIL-ALIGN algorithm we use the following variables for input data and for accumulating data during the execution of the algorithm:

- the variable $Q$ : corresponds to the query bag (the query bacterium) which is a vector of protein sequences.

- the variable $D B$ : corresponds to the bacteria database.

- the variable $S$ : corresponds to a matrix used to store alignment score vectors.

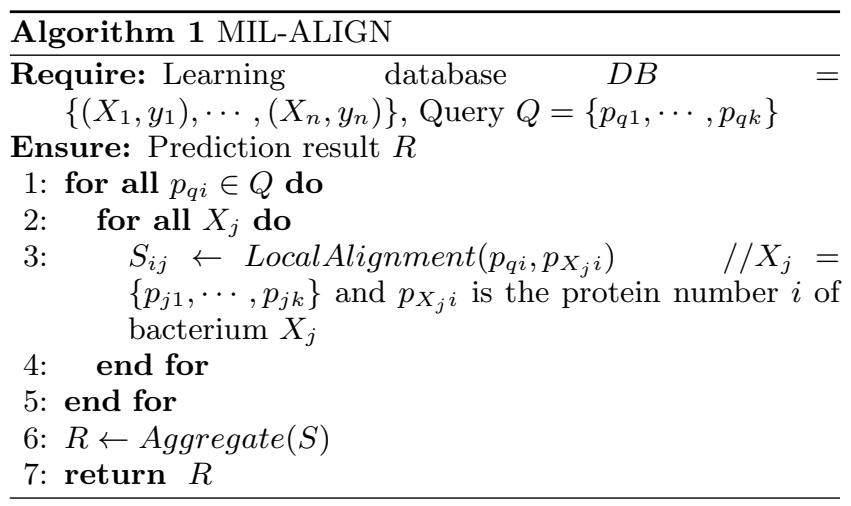
1):

Informally, the algorithm works as follows (see Algorithm

1. For each protein sequence $p_{i}$ in the query bag $Q$, MILALIGN computes the corresponding alignment scores (line 1 to 5 ).

2. Group alignment scores of all protein sequences of query bacterium into a matrix $S$ (line 3). Line $i$ of $S$ corresponds to a score vector of protein $p_{i}$ against all proteins $p_{X_{j} i}$ of $X_{j}$ with $1 \leq j \leq n$. Element $S_{i j}$ corresponds to the alignment score of protein $p_{q i}$ of $Q$ with protein $p_{X_{j} i}$ of bacterium $X_{j}$.
3. Apply an aggregation method to $S$ in order to compute the final prediction result $R$ (line 7). A query bacterium is predicted as IRRB (respectively IRSB) if the aggregation result of similarity scores of its proteins against associated proteins in the learning database is IRRB (respectively IRSB).

\subsection{Experimental environment}

Information on complete and ongoing IRRB genome sequencing projects was obtained from the GOLD database 7]. We initiated our analyses by retrieving orthologous proteins implicated in basal DNA repair in IRRB with fully sequenced genomes. Table 1 presents the used IRRB and IRSB bacteria.

For our experiments, we constructed a training set containing 14 bags ( 8 IRRB and 6 IRSB). Each bag contains at most 30 instances which correspond to proteins implicated in basal DNA repair in IRRB (see Table 2). Protein sequences were downloaded from the FTP site of the curated database SwissProt 1

\section{RESULTS AND DISCUSSION}

\subsection{Experimental techniques}

The computations were carried out on a duo CPU 2.86 $\mathrm{GHz}$ PC with 2 GB memory, operating on Ubuntu Linux. In the classification process, we used the Leave-One-Out (LOO) technique 6 also known as jack-knife test. For each dataset (comprising $n$ bags), only one bag is kept for the test and the remaining part is used for the training. This action is repeated $n$ times. In our context, the leave-one-out is considered to be the most objective test technique compared to the other ones (i.e., hold-out, $n$-cross-validation) as our training set contains a small number of bacteria.

For our tests, we used the BLAST tool [1] for computing local alignments. We implemented two aggregation methods to be used with MIL-ALIGN: the Sum of Maximum Scores method and the Weighted Average of Maximum Scores method.

Sum of Maximum Scores (SMS). For each protein in the query bacterium, we traverse the corresponding line of $S$ which contains the obtained scores against all other bacteria of the training database. The $S M S$ method selects the maximum score among the alignments scores against IRRB (which we call $\max _{R}$ ) and the maximum score among the scores of alignments against IRSB (which we call $\max _{S}$ ). It then compares these scores. If $\max _{R}$ is greater than $\max _{S}$, it adds $\max _{R}$ to the total score of IRRB (which we call $\operatorname{total}_{R}(S)$ ). Otherwise, it adds $\max _{S}$ to the total score of IRSB (which we call $\operatorname{total}_{S}(S)$ ). When all the selected proteins were traversed, the $S M S$ method compares the total scores of IRRB and IRSB. If $\operatorname{total}_{R}(S)$ is greater than $\operatorname{total}_{S}(S)$, prediction refers IRRB. Otherwise, prediction refers IRSB.

Below, we formally define the SMS method:

$$
\operatorname{SMS}(S)= \begin{cases}I R R B, & \text { if } \operatorname{total}_{R}(S) \geq \operatorname{total}_{S}(S), \\ I R S B, & \text { otherwise, }\end{cases}
$$

where

${ }^{1}$ http://www.uniprot.org/downloads 
Table 2: Replication, repair, and recombination proteins related to ionizing-radiation-resistant bacteria

\begin{tabular}{|c|c|c|}
\hline ID & Protein & Function \\
\hline P1 & DNA polymerase III, $\alpha$ subunit & \multirow{5}{*}{ DNA polymerase } \\
\hline P2 & DNA polymerase III, $\epsilon$ subunit & \\
\hline P3 & Putative DNA polymerase III, $\delta$ subunit & \\
\hline $\mathrm{P} 4$ & DNA-directed DNA polymerase & \\
\hline $\mathrm{P} 5$ & DNA polymerase III, $\tau / \gamma$ subunit & \\
\hline P6 & Single-stranded DNA-binding protein & \multirow{6}{*}{ Replication complex } \\
\hline P7 & Replicative DNA helicase & \\
\hline $\mathrm{P} 8$ & DNA primase & \\
\hline P9 & DNA gyrase, subunit B & \\
\hline $\mathrm{P} 10$ & DNA topoisomerase I & \\
\hline P11 & DNA gyrase, subunit A & \\
\hline $\mathrm{P} 12$ & Smf proteins & \multirow{19}{*}{ Other DNA-associated proteins } \\
\hline $\mathrm{P} 13$ & Endonuclease III & \\
\hline $\mathrm{P} 14$ & Holliday junction resolvase & \\
\hline $\mathrm{P} 15$ & Formamidopyrimidine-DNA glycosylase & \\
\hline $\mathrm{P} 16$ & Holliday junction DNA helicase & \\
\hline $\mathrm{P} 17$ & RecF protein & \\
\hline P18 & DNA repair protein & \\
\hline P19 & Holliday junction binding protein & \\
\hline $\mathrm{P} 20$ & Excinuclease $\mathrm{ABC}$, subunit $\mathrm{C}$ & \\
\hline P21 & Transcription-repair coupling factor & \\
\hline P22 & Excinuclease $\mathrm{ABC}$, subunit A & \\
\hline $\mathrm{P} 23$ & DNA helicase II & \\
\hline P24 & DNA helicase RecG & \\
\hline $\mathrm{P} 25$ & Exonuclease SbcC & \\
\hline $\mathrm{P} 26$ & Ribonuclease HII & \\
\hline $\mathrm{P} 27$ & Excinuclease ABC, subunit B & \\
\hline $\mathrm{P} 28$ & A/G-specific adenine glycosylase & \\
\hline P29 & RecA protein & \\
\hline P30 & DNA-3-methyladenine glycosidase II, putative & \\
\hline
\end{tabular}

- $\operatorname{total}_{R}(S)=\sum_{i=1}^{n} \max _{1 \leq j \leq k} \quad S_{i j}$ such that $y_{j}=$ $I R R B$, and

- $\operatorname{total}_{S}(S)=\sum_{i=1}^{n} \max _{1 \leq j \leq k} \quad S_{i j}$ such that $y_{j}=$ IRSB.

Weighted Average of Maximum Scores (WAMS). With the WAMS method, each protein $p_{i}$ has a given weight $w_{i}$. For each protein in the query bacterium, we traverse the corresponding line of $S$ which contains the obtained scores against all other bacteria of the training database. The $W A M S$ method selects the maximum score among the scores of alignments against IRRB (which we call $\max _{R}(S)$ ) and the maximum score among the scores of alignments against IRSB (which we call $\max _{S}(S)$ ). It then compares these scores. If the $\max _{R}(S)$ is greater than $\max _{S}(S)$, it adds $\max _{R}(S)$ multiplied by the weight of the protein to the total score of IRRB and it increments the number of IRRB having a max score. Otherwise, it adds $\max _{S}(S)$ multiplied by the weight of the protein to the total score of IRSB and it increments the number of IRSB having a max score. When all the selected proteins were traversed, we compare the average of total scores of IRRB (which we called $\operatorname{avg}_{R}(S)$ ) and the average of total scores of IRSB (which we called $\operatorname{avg}_{S}(S)$ ). If $\operatorname{avg}_{R}(S)$ is greater than $\operatorname{avg}_{S}(S)$, prediction refers IRRB. Otherwise, prediction refers IRSB.

Below, we formally define the WAMS method:

$$
\operatorname{WAMS}(S)= \begin{cases}I R R B, & \text { if } \operatorname{avg}_{R}(S) \geq \operatorname{avg}_{S}(S), \\ I R S B, & \text { otherwise }\end{cases}
$$

where

- $\operatorname{avg}_{R}(S)=\operatorname{total}_{R}(S) /$ num $_{R}$, and

- $\operatorname{avg}_{S}(S)=\operatorname{total}_{S}(S) /$ num $_{S}$,

and

- $\operatorname{total}_{R}(S)=\sum_{i=1}^{n} \max _{1 \leq j \leq k} S_{i j} \cdot w_{i}$ such that $y_{j}=$ $I R R B$, and

- $\operatorname{total}_{S}(S)=\sum_{i=1}^{n} \max _{1 \leq j \leq k} S_{i j} \cdot w_{i}$ such that $y_{j}=$ IRSB,

where $w_{i}$ is the weight of the protein $p_{i}$.

\subsection{Results}

In order to simulate traditional setting of machine learning in the context of predicting IRRB, we conducted a set of experiments with MIL-ALIGN by selecting just one protein for each bacterium in the training set. Each experiment consists of aggregating alignment scores between a protein sequence of a query bacterium and the corresponding protein sequences of each bacterium in the learning database. We present in Table 3 prediction results with the traditional setting of machine learning. The LOO-based evaluation technique was used to generate the presented results.

As shown in Table 3 we conducted only 22 experiments (with only 22 proteins). This is due to the fact that experiments on proteins which are not expressed at least for 
Table 3: Prediction results with the traditional setting of machine learning

\begin{tabular}{|c|c|c|c|c|c|}
\hline \multirow{2}{*}{ Protein } & \multicolumn{2}{|c|}{ Dataset } & \multirow{2}{*}{ Accuracy (\%) } & \multirow{2}{*}{ Sensitivity (\%) } & \multirow{2}{*}{ Specificity (\%) } \\
\hline & IRRB & IRSB & & & \\
\hline DNA primase & 8 (B1 B2 B3 B4 B5 B6 B7 B8) & 6 (B9 B10 B11 B12 B13 B14) & 85.7 & 87.5 & 83.3 \\
\hline Replicative DNA helicase & 8 (B1 B2 B3 B4 B5 B6 B7 B8) & 6 (B9 B10 B11 B12 B13 B14) & 78.5 & 85.7 & 71.4 \\
\hline DNA topoisomerase I & 8 (B1 B2 B3 B4 B5 B6 B7 B8) & 6 (B9 B10 B11 B12 B13 B14) & 78.5 & 85.7 & 71.4 \\
\hline DNA gyrase, subunit A & $8(\mathrm{~B} 1 \mathrm{~B} 2 \mathrm{~B} 3 \mathrm{~B} 4 \mathrm{~B} 5 \mathrm{~B} 6 \mathrm{~B} 7 \mathrm{~B} 8)$ & 6 (B9 B10 B11 B12 B13 B14) & 71.4 & 75 & 66.6 \\
\hline Endonuclease III & 8 (B1 B2 B3 B4 B5 B6 B7 B8) & 6 (B9 B10 B11 B12 B13 B14) & 71.4 & 70 & 75 \\
\hline Formamidopyrimidine-DNA glycosylase & 8 (B1 B2 B3 B4 B5 B6 B7 B8) & 6 (B9 B10 B11 B12 B13 B14) & 71.4 & 75 & 66.6 \\
\hline RecA Protein & 8 (B1 B2 B3 B4 B5 B6 B7 B8) & 6 (B9 B10 B11 B12 B13 B14) & 64.2 & 66.6 & 60 \\
\hline DNA polymerase III, $\alpha$ subunit & 8 (B1 B2 B3 B4 B5 B6 B7 B8) & 6 (B9 B10 B11 B12 B13 B14) & 57 & 66.6 & 55.5 \\
\hline Excinuclease ABC, subunit A & 8 (B1 B2 B3 B4 B5 B6 B7 B8) & 4 (B9 B10 B11 B12 B13 B14) & 75 & 87.5 & 60 \\
\hline DNA helicase RecG & 5 (B1 B4 B6 B7 B8) & 6 (B9 B10 B11 B12 B13 B14) & 90.9 & 83.3 & 100 \\
\hline Excinuclease $\mathrm{ABC}$, subunit $\mathrm{C}$ & 6 (B1 B2 B3 B5 B7 B8) & 5 (B9 B10 B11 B12 B13) & 81.8 & 100 & 71.4 \\
\hline Transcription-repair coupling factor & 6 (B1 B2 B3 B5 B7 B8) & 5 (B9 B10 B11 B12 B14) & 72.7 & 71.4 & 75 \\
\hline DNA polymerase III, $\tau / \gamma$ subunit & 6 (B2 B3 B4 B5 B6 B7) & 5 (B9 B10 B11 B13 B14) & 72.7 & 80 & 66.6 \\
\hline DNA gyrase, subunit B & 5 (B1 B2 B3 B5 B8) & 6 (B9 B10 B11 B12 B13 B14) & 63.6 & 60 & 66.6 \\
\hline Holliday junction resolvase & $4(\mathrm{~B} 1 \mathrm{~B} 2 \mathrm{~B} 3 \mathrm{~B} 4 \mathrm{~B} 6)$ & 6 (B9 B10 B11 B12 B13 B14) & 70 & 66.6 & 71.4 \\
\hline DNA polymerase III, $\epsilon$ subunit & $6(\mathrm{~B} 1 \mathrm{~B} 2 \mathrm{~B} 3 \mathrm{~B} 4 \mathrm{~B} 6 \mathrm{~B} 8)$ & 3 (B9 B13 B14) & 77.7 & 83.3 & 66.6 \\
\hline Excinuclease ABC, subunit B & 6 (B1 B2 B3 B5 B7 B8) & 3 (B9 B12 B13) & 44.4 & 66.6 & 33.3 \\
\hline RecF protein & 5 (B1 B2 B4 B6 B7) & 3 (B9 B13 B14) & 75 & 80 & 66.6 \\
\hline A/G-specific adenine glycosylase & 7 (B1 B3 B4 B5 B6 B8) & 1 (B13) & 75 & 85.7 & 0 \\
\hline Single-stranded DNA-binding protein & 6 (B1 B4 B5 B6 B7 B8) & 2 (B9 B13) & 50 & 66.6 & 0 \\
\hline Ribonuclease HII & $2(\mathrm{~B} 1 \mathrm{~B} 8)$ & 5 (B9 B10 B11 B12 B13) & 85.7 & 66.6 & 100 \\
\hline DNA-directed DNA polymerase & $4(\mathrm{~B} 2 \mathrm{~B} 3 \mathrm{~B} 5 \mathrm{~B} 6)$ & 1 (B13) & 60 & 75 & 0 \\
\hline
\end{tabular}

one IRRB bacterium and for one IRSB bacterium were not conducted. Results in Table 3 show that the use of our algorithm with just one instance for each bag in the learning database allow good accuracy values especially with some specific proteins. However, almost all results were generated without the whole set of bacteria. In fact, when a protein is not expressed in a specific bacterium, we do not use the bacterium in the learning database. For example, the protein DNA helicase Rec $G$ is expressed for only 11 bacteria ( 5 IRRB and 6 IRSB) from the set of 14 bacteria of the training set (see Table 1).

In order to study the incorrectly classified bacteria with the traditional setting of machine learning, we computed for each bacterium in the learning database, the percentage of experiments that fail to correctly classify the bacterium (see Table 4).

As shown in Table 4, some bacteria present high rates of failed predictions. This means that we fail to correctly predict the phenotype of those bacteria with most proteins. On the other hand, the results illustrated in Table 4 may help to understand some characteristics of the studied bacteria. For example, the Thermus thermophilus SG0.5JP17-16 bacterium presents a high rate of failed predictions $(83.33 \%)$. It mean that in most cases, Thermus thermophilus SG0.5JP1716 is predicted as IRRB. This result shows that Thermus thermophilus SG0.5JP17-16 might allow a strong ability for DNA protection and repair mechanisms and confirm the in vitro results presented in [9], 11] and 10].

In order to study the importance of considering the problem of predicting ionizing-radiation-resistant bacteria as a multiple instance learning problem, we present in Table 5 the experimental results of MIL-ALIGN using a set of proteins to represent the studied bacteria. For each set of proteins and for each aggregation method, we present the accuracy, the sensitivity and the specificity of MIL-ALIGN. We notice that the WAMS aggregation method was used with equally weighted proteins. We used the LOO-based evaluation technique to generate the presented results.

We notice that the use of the whole set of proteins to represent the studied bacteria allows good accuracy accompanied by a high values of sensitivity and specificity especially with the WAMS aggregation method. This can be explained by a good choice of proteins to represents the studied bacteria. For example, with the combination of DNA primase (P8), DNA helicase RecG (P24) and A/G-specific adenine glycosylase (P28) and with the WAMS aggregation method, we have $92.8 \%$ of accuracy, $88.8 \%$ of sensitivity and 100 $\%$ of specificity. We do not exceed these values in all the cases presented in Table 3 This result can be explained by the complementarity between DNA primase (P8), DNA helicase RecG (P24) and A/G-specific adenine glycosylase (P28). In fact, DNA primase (P8) and DNA helicase RecG (P24) present good accuracies in a traditional supervised learning setting (see Table 3 ) and A/G-specific adenine glycosylase (P28) presents the ability to correctly classify bacteria that are incorrectly classified with DNA primase (P8) and DNA helicase RecG (P24).

Table 5 suggests that ionizing resistant radiation is better reflected in three biological processes : (i) synthesis by the DNA primase $(\mathrm{P} 8)$ of small RNA primers for the Okazaki fragments on both template strands at replication forks during chromosomal DNA synthesis; (ii) maintaining genomic stability and integrity by controlling recombination events, and repairing DNA damage by the DNA helicase RecG (P24); and (iii) repair of G-A mispairs and oxidatively damaged form of guanine by MutY (P28).

The high values of specificity presented by MIL-ALIGN show the ability of MIL-ALIGN to identify negative bags (IRSB).

\section{CONCLUSION}

In this paper, we addressed the issue of predicting ionizing-radiation-resistant bacteria (IRRB). We have considered that this problem is a multiple-instance learning problem in which bacteria represent bags and repair proteins of each bacterium represent instances. We have formulated the studied problem and described our proposed algorithm MIL-ALIGN for phenotype prediction in the case of IRRB. By running experiments on a real dataset, we have shown that first results of MIL-ALIGN are satisfactory.

In the future work, we will study the performance of the proposed approach to improve its efficiency. Also, we will study the use of a priori knowledge to improve the efficiency of our algorithm. This a priori knowledge can be used to 
Table 4: Percentage of failed predictions

\begin{tabular}{|c|l|c|}
\hline Phenotype & Bacterium & Rate of failed predictions (\%) \\
\hline \multirow{5}{*}{ IRRB } & Acinetobacter radioresistens SH164 & 15 \\
& Kineococcus radiotolerans SRS30216 & 33.33 \\
& Methylobacterium radiotolerans JCM 2831 & 77.77 \\
& Deinococcus maricopensis DSM 21211 & 0 \\
& Gemmata obscuriglobus UQM 2246 & 47.05 \\
& Deinococcus proteolyticus MRP & 5.88 \\
& Truepera radiovictrix DSM 17093 & 27.77 \\
& Acinetobacter radioresistens SK82 & 11.11 \\
\hline \multirow{5}{*}{ IRSB } & Escherichia coli OP50 & 20 \\
& Neisseria gonorrhoeae MS11 & 6.25 \\
& Neisseria gonorrhoeae PID1 & 0 \\
& Neisseria gonorrhoeae DGI18 & 0 \\
& Pseudomonas putida S16 & 47.61 \\
& Thermus thermophilus SG0.5JP17-16 & 83.33 \\
\hline
\end{tabular}

Table 5: Experimental results of MIL-ALIGN with LOO-based evaluation technique

\begin{tabular}{|c||c||c||c||c|}
\hline \multicolumn{1}{|c||}{ Used proteins } & Aggregation method & Accuracy (\%) & Sensitivity (\%) & Specificity (\%) \\
\hline \multirow{2}{*}{ All proteins } & SMS & 71.39 & 75 & 66.6 \\
& WAMS & 78.5 & 72.7 & 100 \\
\hline \multirow{2}{*}{ DNA Polymerase proteins } & SMS & 71.39 & 75 & 66.6 \\
& WAMS & 78.5 & 77.7 & 80 \\
\multirow{2}{*}{ Replication complex proteins } & SMS & 71.39 & 75 & 66.6 \\
& WAMS & 78.5 & 77.7 & 80 \\
\hline \multirow{2}{*}{ Other DNA-associated proteins } & SMS & 78.5 & 85.7 & 71.4 \\
& WAMS & 85.7 & 87.7 & 100 \\
\hline \multirow{2}{*}{ P8 P24 P28 } & SMS & 92.8 & 88.8 & 100 \\
\hline \multirow{2}{*}{ P6 P7 P8 P24 P28 } & WAMS & 85.7 & 87.7 & 83.3 \\
\end{tabular}

assign weights to proteins during the learning step of our approach. A notable interest will be dedicated to the study of other proteins that can be involved to the high resistance of IRRB to the ionizing radiations and desiccation. In fact, many antioxidant enzymes may play important roles in scavenging free radicals caused by irradiation [5].

\section{Acknowledgement}

This work was supported by the French-Tunisian project: General Directorate for Scientific Research in Tunisia (DGRST)/National Center for Scientific Research in France (CNRS) [IRRB 11/R 14-09]. We would like to thank JanThierry Wegener for English proofreading. We also would like to thank Valerian Callens for technical help.

\section{REFERENCES}

[1] S. F. Altschul, W. Gish, W. Miller, E. W. Myers, and D. J. Lipman. Basic local alignment search tool. Journal of molecular biology, 215(3):403-410, Oct. 1990.

[2] A. Ben-Hur and D. L. Brutlag. Remote homology detection: a motif based approach. In ISMB (Supplement of Bioinformatics), pages 26-33, 2003.

[3] T. G. Dietterich, R. H. Lathrop, and T. Lozano-Pérez. Solving the multiple instance problem with axis-parallel rectangles. Artif. Intell., 89(1-2):31-71, Jan. 1997.
[4] G. Fu, X. Nan, H. Liu, R. Y. Patel, P. R. Daga, Y. Chen, D. Wilkins, and R. J. Doerksen. Implementation of multiple-instance learning in drug activity prediction. BMC Bioinformatics, 13(S-15):S3, 2012.

[5] N. Gao, B.-G. Ma, Y.-S. Zhang, Q. Song, L.-L. Chen, and H.-Y. Zhang. Gene expression analysis of four radiation-resistant bacteria. Genomics Insights, 2:11-22, 062009.

[6] J. Han, M. Kamber, and J. Pei. Data Mining: Concepts and Techniques. Morgan Kaufmann Publishers Inc., San Francisco, CA, USA, 3rd edition, 2011.

[7] K. Liolios, K. Mavromatis, N. Tavernarakis, and N. C. Kyrpides. The Genomes On Line Database (GOLD) in 2007: status of genomic and metagenomic projects and their associated metadata. Nucleic Acids Research, 36(suppl 1):D475-D479, Jan. 2008.

[8] O. Maron and T. L. Pérez. A Framework for Multiple-Instance Learning. In M. I. Jordan, M. J. Kearns, and S. A. Solla, editors, Advances in Neural Information Processing Systems, volume 10, pages 570-576, Cambridge, MA, 1998. The MIT Press.

[9] H. Nishida and M. Nishiyama. Evolution of lysine biosynthesis in the phylum deinococcus-thermus. Int $J$ Evol Biol, 2012, 2012.

[10] N. Ohtani, M. Tomita, and M. Itaya. An extreme thermophile, thermus thermophilus, is a polyploid 
bacterium. J Bacteriol, 192(20):5499-505, 2010.

[11] M. Omelchenko, Y. Wolf, E. Gaidamakova,

V. Matrosova, A. Vasilenko, M. Zhai, M. Daly, E. Koonin, and K. Makarova. Comparative genomics of thermus thermophilus and deinococcus radiodurans : divergent routes of adaptation to thermophily and radiation resistance. BMC Evolutionary Biology, $5(1): 1-22,2005$.

[12] M. V. Omelchenko, Y. I. Wolf, E. K. Gaidamakova, V. Y. Matrosova, A. Vasilenko, M. Zhai, M. J. Daly, E. V. Koonin, and K. S. Makarova. Comparative genomics of Thermus thermophilus and Deinococcus radiodurans: divergent routes of adaptation to thermophily and radiation resistance. BMC Evol Biol, 5:57, 2005.

[13] R. Saidi, S. Aridhi, E. M. Nguifo, and M. Maddouri. Feature extraction in protein sequences classification: a new stability measure. In Proceedings of the ACM Conference on Bioinformatics, Computational Biology and Biomedicine, BCB '12, pages 683-689, New York,
NY, USA, 2012. ACM.

[14] R. Saidi, M. Maddouri, and E. M. Nguifo. Protein sequences classification by means of feature extraction with substitution matrices. BMC Bioinformatics, 11:175, 2010.

[15] H. Sghaier, K. Ghedira, A. Benkahla, and I. Barkallah. Basal dna repair machinery is subject to positive selection in ionizing-radiation-resistant bacteria. $B M C$ Genomics, 9(1):297, 2008.

[16] J. Wang, , and J.-D. Zucker. Solving the multiple-instance problem: A lazy learning approach. In In Proc. 17th International Conf. on Machine Learning, pages 1119-1125. Morgan Kaufmann, 2000.

[17] H. Yamakawa, K. Maruhashi, and Y. Nakao. Predicting types of protein-protein interactions using a multiple-instance learning model. In T. Washio, K. Satoh, H. Takeda, and A. Inokuchi, editors, New Frontiers in Artificial Intelligence, volume 4384 of Lecture Notes in Computer Science, pages 42-53. Springer Berlin Heidelberg, 2007. 